\title{
Luteal ANGPT-TIE system during selected stages of pregnancy, and normal and antigestagen-induced luteolysis in the dog
}

\author{
Aykut Gram, Miguel Tavares Pereira ${ }^{1}$, Alois Boos ${ }^{1}$, Anna T Grazul-Bilska² and \\ Mariusz P Kowalewski ${ }^{1}$ \\ ${ }^{1}$ Institute of Veterinary Anatomy, Vetsuisse Faculty, University of Zurich, Zurich, Switzerland, ${ }^{2}$ Department of Animal \\ Sciences, North Dakota State University, Fargo, North Dakota, USA, \\ Correspondence should be addressed to A Gram; Email: aykutgram@gmail.com
}

\begin{abstract}
Rapid establishment of a vascular network is essential for normal functionality of the corpus luteum (CL). The early luteal phase is associated with increased expression of the VEGF system in canine CL. Acting in synchrony with angiopoietins (ANGPTs), VEGF system plays major roles in stabilization of blood vessels. However, the expression of the ANGPT system has not yet been investigated in the dog. Therefore, here, we investigated the luteal expression of ANGPT1, -2, and of their receptors TIE1 and -2, in pregnant dogs at selected time points during pregnancy and at normal and antigestagen-induced luteolysis. Additionally, luteal cells from early $\mathrm{CL}$ were incubated with PGE2 and its effects on the ANGPT system were assessed. Whereas the luteal ANGPT1 was stable until midgestation, TIE1 was elevated post-implantation, their expression decreased toward prepartum luteolysis. The ANGPT2- and TIE2mRNA did not vary during pregnancy. The ANGPT2/ANGPT1 ratio was elevated during prepartum luteolysis. PGE2 increased ANGPT2, but suppressed ANGPT1 levels. None of the ANGPT-system members was affected by antigestagen treatment in midpregnancy. Localization of ANGPT1 was predominantly found in the tunica intima and media of vessels and ANGPT2 stained strongly in luteal cells. Both ANGPTs were localized in macrophages. TIE1 stained in the vascular tunica media, in luteal cells and macrophages, whereas TIE2 was colocalized with ANGPT1 in vascular components. In conclusion, high expression of ANGPT1 during the increased presence of VEGFA in early canine CL implies its contribution to vascular network development. The upregulation of the ANGPT2/ANGPT1 ratio during prepartum luteolysis indicates involvement of the ANGPT system in PGF2 $\alpha$-mediated vascular destabilization.

Reproduction (2018) 156 451-461
\end{abstract}

\section{Introduction}

The corpus luteum $(\mathrm{CL})$ is a rapidly growing, transient endocrine gland, relatively small in the dog, that arises within the ovary following ovulation by differentiation of the remnants of granulosa and theca cells into luteal cells (Channing et al. 1980, Smith et al. 1994, Fraser \& Wulff 2003, Kowalewski et al. 2015). During the early luteal phase, rapid angiogenesis and vasculogenesis are fundamental requirements for the proliferation, development and maintenance of CL (Stouffer \& Hannebold 2015). An integral mediator of both processes is vascular endothelial growth factor A (VEGFA) acting through its respective receptors (VEGFR1/FIt-1, VEGFR2/ Flk-1), which are referred to together as the VEGF system (Ferrara \& Davis-Smyth 1997, Cebe-Suarez et al. 2006, Stuttfeld \& Ballmer-Hofer 2009). The function of this system is complemented, and under certain conditions coordinated, by angiopoietins (ANGPTs) and their tyrosine kinase receptors, TIE1 and TIE2, respectively. ANGPTs affect development of vascular networks by regulating the stability and/or disruption of endothelial cells, the growth of which is stimulated by VEGFs (Hanahan 1997, Brindle et al. 2006). In particular, ANGPT2 is a potent disruptor of endothelial cells (Brindle et al. 2006, Felcht et al. 2012) and allows VEGFA to induce proliferation, invasion and, thereby, formation of new blood vessels in developing CL during the early luteal phase (Fraser \& Wulff 2003). At the mid-luteal phase, in the presence of VEGFA, ANGPT1 supports vessel integrity and inhibits vascular leakage, thereby promoting survival of blood vessels (Hazzard \& Stouffer 2000, Wulff et al. 2000, Sugino et al. 2005). Finally, at the end of the CL life span, during the physiological processes of luteal regression or luteolysis, in the absence of angiogenic factors and in particular of VEGFA, the luteal microvasculature is disrupted by ANGPT2 (Hazzard \& Stouffer 2000, Wulff et al. 2000, Sugino et al. 2005). Apparently, thus, establishment and maintenance of $C L$ depend not only on vasculogenesis and angiogenesis, but also on vascular structural stability, being essential for an adequate supply of luteal 
tissues with oxygen and nutrients, allowing the delivery of progesterone (P4), estrogens and other endocrine factors into the circulation.

In dogs, CLs exhibit an inherent life span, and there is no other major source of circulating P4 in this species during pregnancy and non-pregnant cycles (Concannon et al. 1989, Hoffmann et al. 1994, Nishiyama et al. 1999). This emphasizes the role of an adequate blood supply for the provision of circulating luteal steroids in this species. As in other mammalian species, the canine $C L$ is formed immediately after ovulation, in a process which is associated with intense angiogenesis (Hoffmann et al. 2004a, Gram et al. 2015a). As recently reported from our research, a high vascularization rate and increased blood supply within canine CL during the early stages of its development are mirrored in increased availability of the VEGF system and vasodilatory endothelin receptor B (ETB) (Gram et al. $2015 a, b)$. During early diestrus, prostaglandins (PG), in particular PGE2 acting as a luteotropic factor, appear to be involved in the establishment of canine $\mathrm{CL}$ function (Kowalewski et al. 2013, 2015, Janowski et al. 2014). Interestingly, PGE2 has been shown to increase expression of $E T B$ in isolated canine primary luteal cells (Gram et al. 2015b). These findings show that PGs, in particular PGE2, participate in regulating luteal vascular function in the dog. As evidenced from investigations on the expression of type I membrane glycoprotein endoglin (END), the vascularization rate slows down in the mid-luteal phase in both CL of pregnancy and in nonpregnant dogs (Hoffmann et al. 2004a, Nowaczyk et al. 2017). Interestingly, in pregnant dogs, concomitantly with increasing circulating concentrations of luteolytic PGF2 $\alpha$, an upregulation of the vasoconstrictive endothelin receptor ETA is observed during prepartum luteolysis (Gram et al. 2015b). This clearly indicates the involvement of vascular endothelial functionality at the time of cessation of luteal function during the onset of parturition in the dog. A possible contribution of the inflammatory and immune responses was recently indicated from our analysis of luteal transcripts during prepartum luteolysis (Zatta et al. 2017). Contrasting with the situation observed in pregnant animals, there is a slow luteal regression in the absence of pregnancy, obviously devoid of functional alteration of the vascular bed (Hoffmann et al. 2004b, Gram et al. 2015b).

Nevertheless, in contrast with other domestic animals, and despite the undisputed critical importance of the ANGPT system in regulating vascular stabilization and remodeling, until now its expression in the canine $\mathrm{CL}$ of pregnancy has not been investigated. Therefore, based on the hypothesis that the ANGPT system is involved in regulation of canine CL function, and in order to expand our understanding about the potential role of vascular growth factors in canine $\mathrm{CL}$, here, the following experiments were performed: (1) a descriptive study addressing the protein and mRNA expression of
ANGPT1, -2 and their receptors TIE1 and TIE2 in canine $\mathrm{CL}$ at selected time points during pregnancy and at normal prepartum luteolysis; (2) an in vivo approach investigating P4-mediated effects in bitches, in which luteal function was interrupted during mid-gestation with the antigestagen aglepristone and (3) an in vitro functional approach investigating the role of PGE2 in regulating expression of the ANGPT system in canine primary luteal cells isolated from early $\mathrm{CL}$.

\section{Materials and methods}

\section{Experimental design, collection of luteal tissue and preservation}

All luteal tissues were derived from pregnant, healthy, mixed breed dogs and were used in our previous studies (Kowalewski et al. 2013, Gram et al. 2015a,b). All experimental procedures were performed in accordance with animal welfare legislation and approved by the respective authorities of the Justus-Liebig University (permit no. II 25.3-19c20-15c GI 18/14 and VIG3-19c-20/15c GI 18,14), Giessen, Germany and the University of Ankara (permit no. Ankara 2006/06), Ankara, Turkey.

Briefly, CLs were collected following ovariohysterectomy (OHE) at different stages of pregnancy: early luteal phase (pre-implantation, days $8-12, n=5$, and post-implantation, days $18-25, n=5)$, mid-luteal phase (mid-gestation, days $35-40, n=5)$ and prepartum luteolysis $(n=3)$. Additionally, aglepristone (Alizine, Virbac, Bad Oldesloe, Germany; $10 \mathrm{mg} /$ kg bw; $2 \times / 24$ h apart), P4 receptor (PGR) antagonist was used for termination of pregnancies in mid-pregnant dogs (days 40-45 of pregnancy; $n=10$ ) and OHE was performed $24 \mathrm{~h}$ $(n=5)$ and $72 \mathrm{~h}(n=5)$ after the second treatment.

Dogs 2-8 years of age were mated 2 days after ovulation (determined as the day when P4 for the first time exceeded levels of at least $5 \mathrm{ng} / \mathrm{mL}$ in peripheral blood). The day of mating was considered as day 0 of pregnancy. The pre-implantation stage of pregnancy was detected by observation of embryos in uterine flushes. Prepartum luteolysis samples were obtained by assaying $\mathrm{P} 4$ concentration in blood serum starting on day 58 of pregnancy. Serum P4 levels were then measured every $6 \mathrm{~h}$ until a continuous decrease (below $2-3 \mathrm{ng} / \mathrm{mL}$ ) was detected in 3 consecutive measurements, and then OHE was performed.

After collection, CLs were carefully dissected, cleared of surrounding tissues and fixed with $10 \%$ neutral PBS-buffered formalin for $24 \mathrm{~h}$ at $+4{ }^{\circ} \mathrm{C}$, and then incubated in PBS for 1 week, dehydrated through an ethanol series and embedded in paraffin wax for immunohistochemistry (IHC). For total RNA extraction, tissue samples were incubated in RNAlater (Ambion Biotechnology $\mathrm{GmbH}$, Wiesbaden, Germany) for $24 \mathrm{~h}$ at $+4^{\circ} \mathrm{C}$ and then frozen at $-80^{\circ} \mathrm{C}$ for later evaluation of gene expression.

\section{Primary luteal cell cultures}

Primary luteal cells were isolated from CLs of bitches $(n=15)$ that underwent routine OHE in the Department of Small Animal Reproduction, Vetsuisse Faculty, University of Zurich. All dogs 
were clinically healthy and were in the early diestrus stage of the reproductive cycle (7-14 days after clinical signs of estrus had ceased). All tissue materials were used in our previous study determining the role of PGE2 in regulating canine luteal steroid acute regulatory STAR protein expression and steroidogenesis (Kowalewski et al. 2013, Gram et al. 2015b). In those previous experiments, the steroidogenic capability of isolated cells and their responsiveness to stimulation with PGE2 were demonstrated. In the present study, using the available mRNA, the impact of PGE2 on gene expression of ANGPT system family members (i.e., ANGPT1, ANGPT2, TIE1 and TIE2) was investigated in these cells. In brief, luteal cells were isolated following tissue dissociation with $0.15 \%$ collagenase (Sigma-Aldrich Chemie $\mathrm{GmbH}$ ). Crude cell and remnant tissue suspension was filtered through a $75 \mu \mathrm{m}$ cell strainer (BD Biosciences, Basel, Switzerland). This was followed by washing steps with PBS. Freshly isolated cells were then suspended in culture medium (DMEM/F12, $\mathrm{pH} 7.2-7.4$, $10 \%$ heat-inactivated FBS, $100 \mathrm{U} / \mathrm{mL}$ penicillin and $100 \mu \mathrm{g} /$ $\mathrm{mL}$ streptomycin, 1\% ITS (Insulin-Transferrin-Selenium); all from Chemie Brunschwig, AG, Basel, Switzerland), transferred into six-well plates and cultured in an incubator $\left(37^{\circ} \mathrm{C}, 5 \%\right.$ $\mathrm{CO}_{2}$ ). Experiments were performed with cells that had reached 70-80\% confluence. Importantly, non-trypsinized (i.e., passage 0 ) cells were used for in vitro experiments performed in serum-free medium. Cells were treated with $20 \mu \mathrm{M}$ PGE2 (Sigma-Aldrich Chemie $\mathrm{GmbH}$ ), which was determined to be an effective dosage in functional assays with canine proximal STAR promoter bearing intact CAMP/PKA-responsive elements (Kowalewski et al. 2013). Additionally, P4 concentrations were determined as a measure of steroidogenic activity in PGE2-treated cells (Kowalewski et al. 2013). Treatments were done for $6 \mathrm{~h}$, a time course, which is needed to induce a full steroidogenic response at the level of STAR-dependent steroidogenesis (Kowalewski et al. 2013). With this approach, PGE2 was determined to be an activator of STAR gene expression in canine primary luteal cells at levels that are similar to those observed with dbcAMP treatment (Kowalewski et al. 2013).

\section{RNA isolation, reverse transcription and semi- quantitative real-time (TaqMan) PCR}

Total RNA was extracted from $\mathrm{CL}$ samples and luteal cells using TRIzol reagent (Invitrogen) according to the manufacturer's protocol and our previously published protocols (Kowalewski et al. 2006b, 2011a). A NanoDrop 2000C spectrophotometer (Thermo Fisher Scientific AG) was used to check the quality and quantity of isolated total RNA. Following DNase treatment with RQ1 RNase-free DNase (Promega, Dübendorf, Switzerland), for removal of genomic DNA, all RNA samples were reverse transcribed (RT) into cDNA using random hexamers as primers in the presence of other RT reagents (Applied Biosystems by Thermo Fisher). The ABI PRISM 7500 Sequence Detection System (Applied Biosystems) was used to perform semi-quantitative RealTime (TaqMan) PCR. The amplification conditions were as follows: denaturation at $95^{\circ} \mathrm{C}$ for $10 \mathrm{~min}$, followed by 40 cycles, $95^{\circ} \mathrm{C}$ for $15 \mathrm{~s}$ and $60^{\circ} \mathrm{C}$ for $60 \mathrm{~s}$, each. As a control, all experiments were run with all necessary components of the amplification reaction except for reverse transcriptase to check for any potential genomic DNA contamination (the so-called minus-RT control). Additionally, a non-template control was performed by using water instead of cDNA.

Self-designed primers and TaqMan Probes for ANGPT system members were selected using the Primer Express Software $v$ 2.0 (Applied Biosystems) and were bought from Microsynth (Balgach, Switzerland). Primers and 6-carboxyfluorescein (6-FAM)- and 6-carboxytetramethylrhodamine (TAMRA)labeled TaqMan probes are listed in Table 1. Amplifications of all genes were done in duplicates. Three different reference genes, GAPDH, CYCLOPHILIN $A$ and $\beta$-ACTIN were used to normalize gene expression profiles.

For CYCLOPHILIN A (Prod. No. Cf03986523_gH) and $\beta$-ACTIN (Prod. No.184 Cf03023880_g1), commercially available canine-specific TaqMan Gene Expression Assays were used, purchased from Applied Biosystems. The comparative CT $(\Delta \Delta \mathrm{CT})$ method was used to calculate relative gene expression levels of each target gene according to the protocol of the manufacturer of the ABI Prism 7500 (Applied Biosystems). The sample with the lowest expression for each target gene was used as a calibrator. The amplification efficiency of the PCR assays was calculated by the CT slope method ensuring approximately $100 \%$ reaction efficiency.

Following quantification of ANGPT1 and ANGPT2 mRNA expression with the comparative $C T$ method using three different reference genes (GAPDH, CYCLOPHILIN $A$ and $\beta$-ACTIN) as normalizers, and applying the same Ct values and calibrators, relative gene expression values for both genes were divided by each other to calculate the ANGPT2/ANGPT1 mRNA ratio.

\section{Molecular cloning of canine TIE1 and TIE2 sequences}

Canine-specific TIE1 and TIE2 (TEK) sequences have not been characterized previously, and were available from GenBank only as predicted sequences. In order to provide templates for the subsequent PCR reactions, molecular cloning and sequencing were performed following our previously published protocol (Kowalewski et al. 2006b, 2011b, Kautz et al. 2014). Therefore, using available predicted sequences, gene-specific primers were designed that allow us to amplify partial CDS of canine-specific TIE1 and TIE2 (TEK). Following DNase treatment (Promega) and RT reactions, a conventional hot start PCR reaction was done using the GeneAmp Gold RNA PCR Kit (Applied Biosystems) following the manufacturer's protocol. Primers, purchased from Microsynth, were as follows: TIE1, forward: 5'-AGA GCC GGG TCT TGG AGA-3', reverse: 5'-ATG CCC GCG TAA GTG AAG TT-3'; TIE2 forward: 5'-AAT CAA CGG TGC CTA TTT CTG T-3', reverse: 5'-CAG ACC CTG CCA ACC TGT G-3'. PCR reactions were run with the annealing temperature set at $60^{\circ} \mathrm{C}$ for both TIE1 and TIE2. Negative controls were as for TaqMan PCR. Specific amplicons of $601 \mathrm{bp}$ and $604 \mathrm{bp}$ for TIE1 and TIE2, respectively, were generated, separated in $2 \%$ ethidium bromide-stained agarose gel and purified using the Qiaex II gel extraction system (Qiagen $\mathrm{GmbH}$ ). Following subcloning of PCR products into the pGEM-T vector (Promega), they were transformed and amplified in XL1 Blue competent cells (Stratagene, La Jolla, 
Table 1 List of self-designed primers used for Real-Time (TaqMan) PCR.

\begin{tabular}{|c|c|c|c|}
\hline Gene & Primer sequence & Product length (bp) & Accession number \\
\hline ANGPT1 & $\begin{array}{l}\text { F: 5'-AAG TCG GAG ATG GCC CAG ATA-3' } \\
\text { R: 5'-CAG TCT GAG AGA GGA GGC TGG TT-3' } \\
\text { T: 5'-CAC ACG GCC ACC ATG CTC GAG ATA G-3' }\end{array}$ & 91 & AB192412.2 \\
\hline ANGPT2 & $\begin{array}{l}\text { F: 5'-ACG AGG CGT ACT CGC TGT ATG-3' } \\
\text { R: 5'-CCT GGT TGG CTT ATG CTG CTT-3' } \\
\text { T: 5'-ATT CAC CTT AAA GGA CTT ACA GGG ACG GCC-3' }\end{array}$ & 118 & AB256019.1 \\
\hline TIE1 & $\begin{array}{l}\text { F: 5'-GAC CTG TGC TGA GCT CTA TGA GAA-3' } \\
\text { R: 5'-ACG CAT CAG CTC GTA CAC TTC A-3' } \\
\text { T: 5'-TAC CGC ATG GAA CAG CCT CGA AAC TG-3' }\end{array}$ & 91 & MG932084 \\
\hline TIE2 & $\begin{array}{l}\text { F: 5'-TCA GCC AGG TAC ATA GGA GGA AAC-3' } \\
\text { R: 5'-GTT ACA TTC AGG TCC CCA CTT CTG-3' } \\
\text { T: 5'-TCT TCA CCT CGG CCT TCA CCA GGC T-3' }\end{array}$ & 96 & MG932085 \\
\hline GAPDH & $\begin{array}{l}\text { F: 5'-GCT GCC AAA TAT GAC GAC ATC A-3' } \\
\text { R: 5'-GTA GCC CAG GAT GCC TTT GAG-3' } \\
\text { T: 5'-TCC CTC CGA TGC CTG CTT CAC TAC CTT-3' }\end{array}$ & 75 & AB028142.1 \\
\hline
\end{tabular}

F, forward; R, reverse; T, TaqMan probe.

CA, USA). Plasmids were isolated and purified using Pure Yield Plasmid MidiPrep System (Promega) and were sent for commercial sequencing on both strands with T7 and Sp6 primers (Microsynth). Finally, the sequences obtained were submitted to GenBank with the following accession numbers: TIE1 GenBank acc. Nr. MG932084, and TIE2 (TEK) GenBank acc. Nr. MG932085. BLAST analysis revealed 93\% identity with respective fragments of human and sheep TIE 1 sequences. There was a $92 \%$ identity with fragments of human and sheep for the TIE2 sequence.

\section{Immunohistochemistry}

The indirect immunoperoxidase immunohistochemistry (IHC) assay was performed on tissue samples obtained from pregnant dogs using our previously published protocol (Kowalewski et al. 2006a,b). Briefly, formalin-fixed, paraffinembedded CL samples were sectioned at 2-3 $\mu \mathrm{m}$ thickness and mounted onto SuperFrost microscope slides (Menzel-Glaeser, Braunschweig, Germany). Following deparaffinization with xylene and rehydration in a graded ethanol series, tissue sections were heated in a microwave oven at 560W for $15 \mathrm{~min}$ in $10 \mathrm{mM}$ citrate buffer $\mathrm{pH} 6.0$ for antigen retrieval. Nonspecific antibody reactivity was blocked by incubating slides with $10 \%$ normal serum from the same species in which the secondary antibody was produced. Then, slides were incubated with primary antibodies overnight at $4^{\circ} \mathrm{C}$. The dilutions of primary antibodies used in this study were 1:50 for canine-specific, rabbit polyclonal anti-ANGPT1 antibody (PAA008Ca01, Cloud-Clone Corp., TX, USA); 1:200 for rabbit anti-mouse ANGPT2 polyclonal antibody (TA343276, OriGene Technologies Inc., Rockville, MD, USA); 1:100 for rabbit anti-human TIE1 (H-180) polyclonal antibody (sc9025, Santa Cruz Biotechnology Inc.) and 1:200 for goat anti-human TIE2 (A-14) polyclonal antibody (sc-31268, Santa Cruz Biotechnology). Macrophages were identified by using polyclonal rabbit anti-human MHCII antibody (orb101661, Biorbyt Limited, Cambridge, UK, dilution 1:200). As an isotype control, non-immune IgGs of the same species as the primary antibody were applied instead of primary antibodies, using the same protein concentration. Afterward, sections were rinsed with IHC buffer containing 0.3\% Triton $\mathrm{X} \mathrm{pH} \mathrm{7.2-7.4} \mathrm{(0.8} \mathrm{mM}$ $\mathrm{Na}_{2} \mathrm{HPO}_{4}, 1.47 \mathrm{mM} \mathrm{KH}{ }_{2} \mathrm{PO}_{4}, 2.68 \mathrm{mM} \mathrm{KCl}, 137 \mathrm{mM} \mathrm{NaCl}$ ) and incubated with biotin-labeled secondary antibodies at 1:100 dilution each. These were horse anti-goat IgG BA-9500 and goat anti-rabbit IgG BA1000 (both purchased from Vector Laboratories Inc., Burlingame, CA, USA). Subsequently, sections were washed, incubated with a streptavidinperoxidase Vectastain ABC kit (Vector Laboratories) for $30 \mathrm{~min}$ and then antigen-antibody complexes were visualized using the Liquid DAB+ substrate kit (Dako Schweiz AG). Counterstaining was performed using hematoxylin. Finally, sections were dehydrated in a graded ethanol series and xylene and mounted in Histokit 180 (Assistant, Osterode, Germany). Slides were assessed qualitatively.

\section{Statistical analyses}

Since the data obtained in our current study did not show a normal distribution, logarithmic transformations were performed for normalization. For statistical calculation of differences in the expression of ANGPT-system members between different stages of pregnancy (time-dependent effect), one-way ANOVA was performed. This, in case of $P<0.05$, was followed by the Tukey-Kramer multiple comparisons test, in which all groups were compared with each other. In order to assess the effects of the P4 receptor antagonist (aglepristone) on expression of the ANGPT system, first a global comparison was performed by applying one-way ANOVA. Next, using the mid-pregnant group as a non-treated control, Dunnett's multiple comparison test was performed to show expression of the ANGPT system in CL of antigestagen-treated dogs. In this case, the results show the $n$-fold change in each target gene mRNA compared with its expression at mid-gestation in a nontreated control. The effect of PGE2 on expression of ANGPT system members in cell cultures was calculated using an unpaired two-tailed Student's $t$-test. For all analyses, numerical data are presented as geometric means $\mathrm{Xg} \pm$ geometric standard deviation (s.D.). All statistical calculations were performed using the statistical software program GraphPad 3.06 (GraphPad Software). The level of significance was considered as $P<0.05$. 


\section{Results}

\section{Quantification of gene expression}

Expression of ANGPT1, -2 and their receptors TIE1 and TIE2 was detectable in all CL samples at every pregnancy stage investigated in the present study. The ANGPT1 mRNA showed a time-dependent expression profile during pregnancy (ANOVA, $P<0.0001$ ) (Fig. 1A). It was more or less constantly expressed from pre-implantation until mid-gestation $(P>0.05)$; however, it decreased significantly $(P<0.01)$ during prepartum luteolysis compared with all previous stages of pregnancy (Fig. 1A). The expression of ANGPT2 mRNA did not differ significantly over time (ANOVA, $P=0.3$ ) (Fig. 1B). The ANGPT2/ANGPT1 ratio was also assessed and was time related (ANOVA, $P=0.001$ ). Thus, whereas it was unaffected from early until mid-gestation $(P>0.05)$, it increased significantly $(P<0.05)$ during prepartum luteolysis compared with all previous stages (Fig. 1C).

As for ANGPT receptors, TIE1 mRNA levels changed significantly over time (ANOVA, $P=0.02$ ).
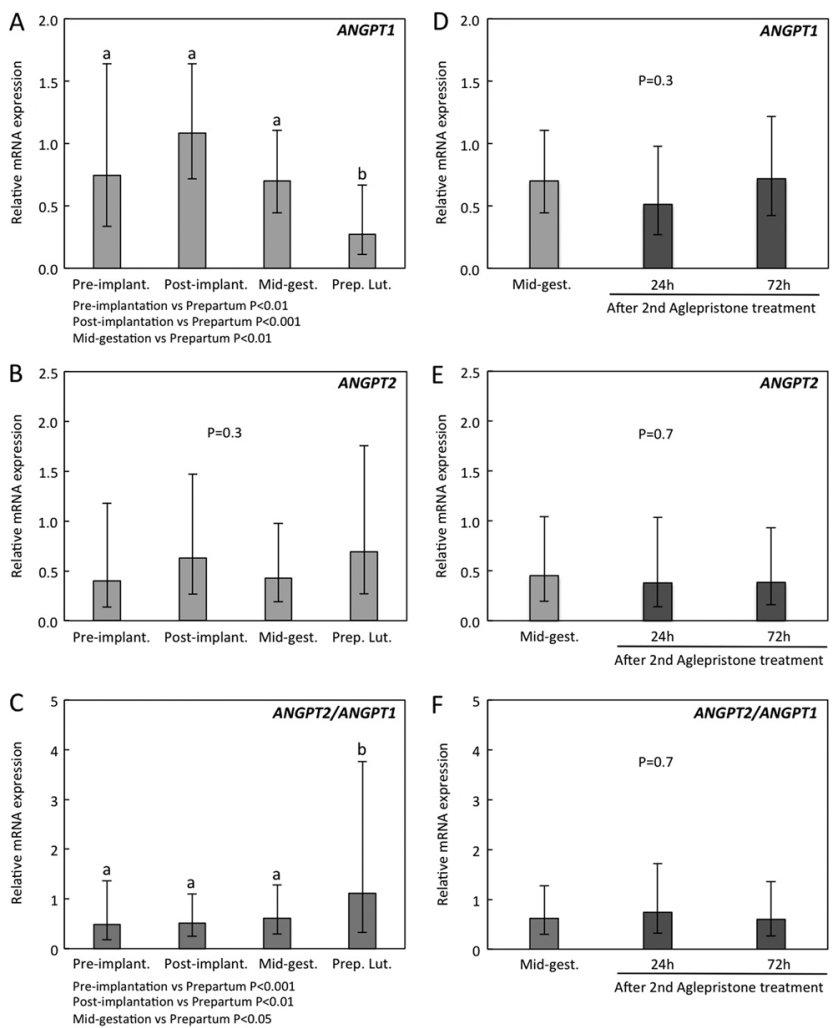

Figure 1 Expression of ANGPT1, ANGPT2 and the ANGPT2/ ANGPT1 mRNA ratio as determined by Real-Time (TaqMan) PCR $(\mathrm{Xg} \pm$ S.D.) in canine $\mathrm{CL}$ at selected stages of pregnancy (A, B and C) and during antigestagen (aglepristone)-induced luteolysis in mid-pregnant dogs (D, E and F; compared with the mid-gestation group used as non-treated control). Pre-implant. = pre-implantation stage of pregnancy, Post-implant. $=$ post-implantation stage of pregnancy, Mid-gest. $=$ mid-gestation stage of pregnancy, Prep. Lut. $=$ normal prepartum luteolysis.
Whereas its expression did not vary strongly from preimplantation until mid-gestation, it was significantly lower at prepartum luteolysis compared with the postimplantation stage $(P<0.05)$ (Fig. 2A). Similarly, as for ANGPT2, the abundance of TIE2 transcripts did not differ significantly over time (ANOVA, $P=0.1$ ) (Fig. 2B).

With regard to the preterm luteolysis/abortion groups, the expression of all ANGPT family members and the ANGPT2/ANGPT1 ratio remained unaffected by treatment with an antigestagen, both $24 \mathrm{~h}$ and $72 \mathrm{~h}$ after the second application of aglepristone, compared with the mid-gestation group (ANOVA: ANGPT1, $P=0.3$; ANGPT2, $P=0.7 ;$ ANGPT2/ANGPT1, $P=0.7 ;$ TIE1, $P=0.3$; TIE2, $P=0.8$ ) (Figs 1D, E, F and 2C, D).

\section{Immunolocalization of the ANGPT system in CL of pregnant dogs}

Expression of ANGPT1, -2 and their receptors TIE1 and TIE2 was detectable at the protein level in the $\mathrm{CL}$ at all selected time points during canine pregnancy. Whereas ANGPT1 was clearly detectable in vascular tunica media and tunica intima (endothelial cells) (Fig. 3A, B and C), only weak or no signals were observed in steroidogenic luteal cells (Fig. 3A, B, C and D). Additionally, some signals were localized in interstitial cells in proximity to luteal vessels, which were identified as immune system-derived macrophages (Nowaczyk et al. 2017). At mid-gestation and during prepartum luteolysis, signal intensities appeared weaker and remained primarily localized in
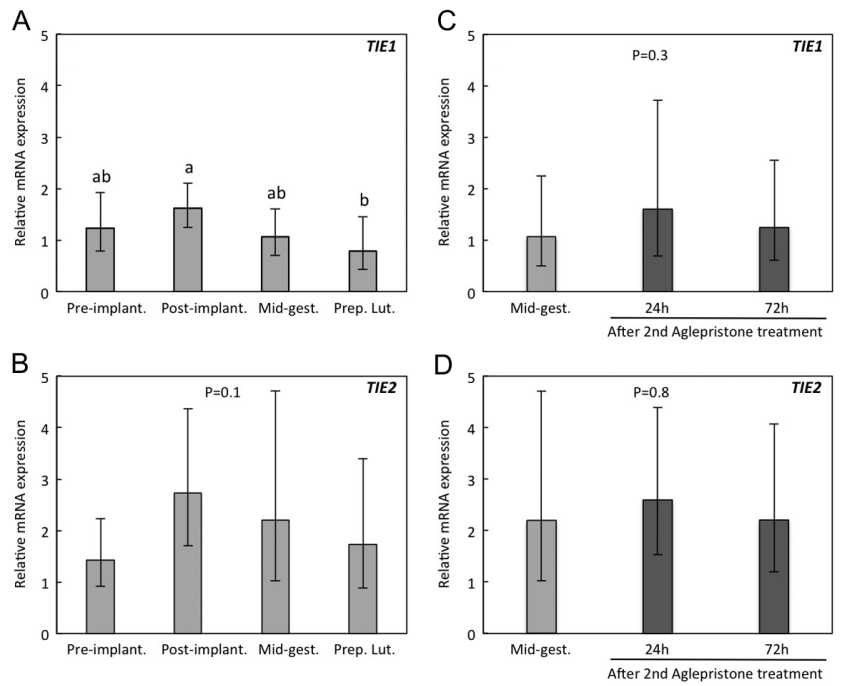

Figure 2 Expression of TIE1 and TIE2 as determined by Real-Time (TaqMan) PCR (Xg \pm S.D.) in canine $\mathrm{CL}$ at selected stages of pregnancy (A and B) and during antigestagen (aglepristone)-induced luteolysis in mid-pregnant dogs (C and D; compared with the mid-gestation group used as non-treated control). Bars with different letters differ at $P<0.05$. Pre-implant. $=$ pre-implantation stage of pregnancy, Post-implant. $=$ post-implantation stage of pregnancy, Mid-gest. $=$ midgestation stage of pregnancy, Prep. Lut. = normal prepartum luteolysis. 


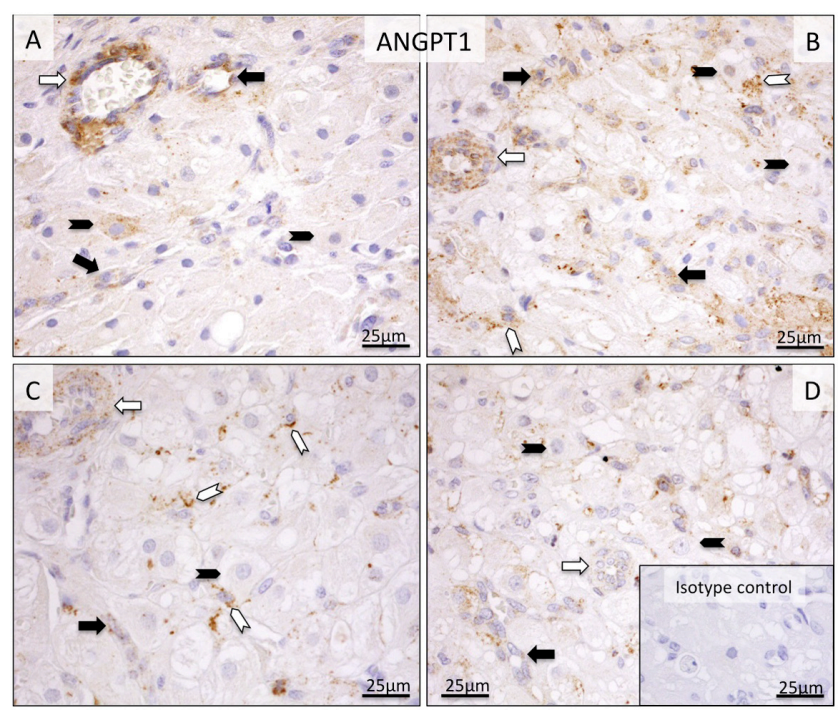

Figure 3 Immunohistochemical localization of ANGPT1 in canine CL at selected time points throughout pregnancy: pre-implantation (A), post-implantation (B), mid-gestation (C) and prepartum luteolysis (D). Strong ANGPT1 signals were detected in the tunica media of vessels (open arrows in A, B, C and D), endothelial cells (solid arrows in A, $\mathrm{B}, \mathrm{C}$ and D) and in luteal macrophages (open arrowheads in B and C). Weaker or no signals are detected in luteal cells (solid arrowheads in A, B, C and D). No background staining is detected in the isotype control (inset in D).

vascular structures and immune cells (Fig. 3C and D). In particular, during prepartum luteolysis, luteal cells appeared negative for ANGPT1. Conversely, ANGPT2 was strongly represented throughout the $\mathrm{CL}$ life span. Signals were seen in luteal cells and macrophages. The staining intensity varied between cells within the same population (Fig. 4A, B, C and D). No staining was observed in the vascular bed (Fig. 4A, B, C and D). Detection of $\mathrm{MHCll}$ was performed in order to more easily differentiate cell types within canine $\mathrm{CL}$ (shown on consecutive sections in Fig. $4 \mathrm{E}$ and $\mathrm{F}$ ). $\mathrm{MHCll}$ is a marker of antigen-presenting cells, shown recently to be predominantly localized in macrophages localized close to luteal blood vessels in canine CL (Nowaczyk et al. 2017). The distribution patterns of TIE1 were similar to those observed for ANGPT2. Thus, signals were present in luteal cells and macrophages (Fig. 5A, B, C and D). Those signals localized in luteal cells appeared, however, weaker than those observed for ANGPT2. Additionally, staining was observed in leukocytes localized in blood vessels (Fig. 5A and C). Relatively weak staining was noted for TIE1 in tunica media and tunica intima of vessels (endothelial cells) (Fig. 5A, B and D). Signals were present in all stages investigated, but intensities appeared weaker during prepartum luteolysis (Fig. 5D). As for TIE2 expression, it was predominantly localized in tunica media of larger vessels and intima of smaller vessels and capillaries (Fig. 6A, B, C and D). Sporadically, signals

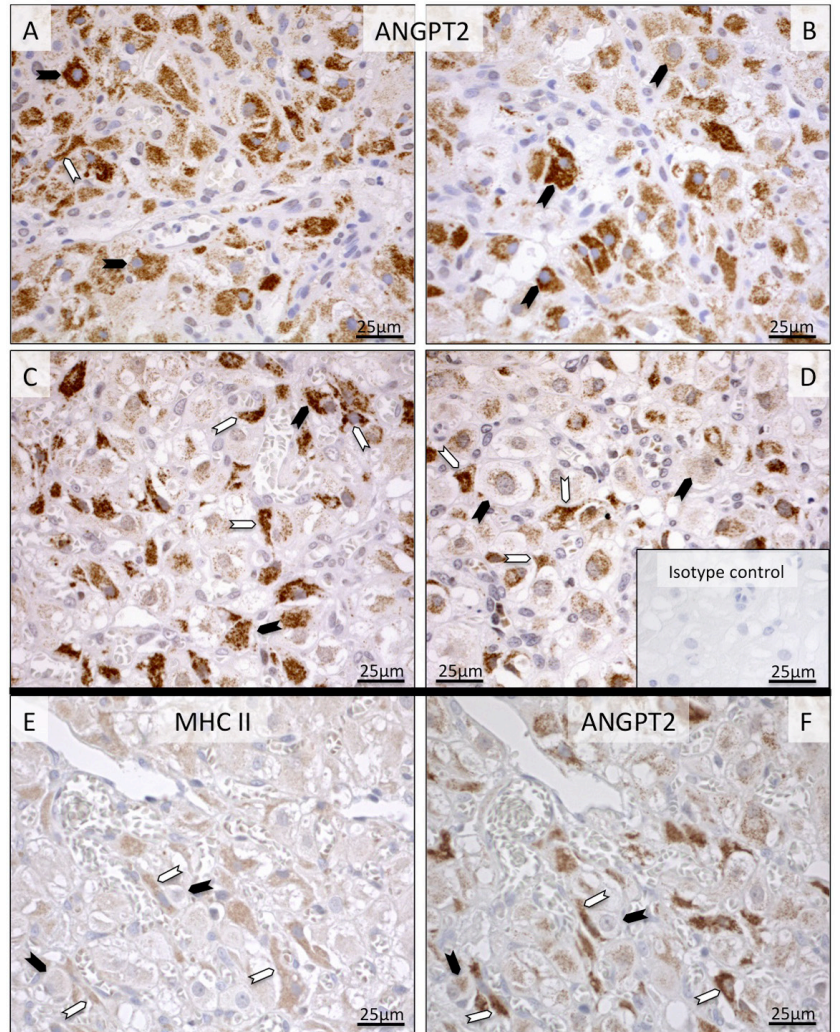

Figure 4 Immunohistochemical localization of ANGPT2 in canine CL at selected time points throughout pregnancy: pre-implantation $(A)$, post-implantation (B), mid-gestation (C) and prepartum luteolysis (D). Additionally, consecutive sections were stained against MHCII (E) and ANGPT2 (F, shown at mid-gestation). The detection of MHCII was performed in order to more easily differentiate cell types within canine CL. Solid arrowheads indicate luteal cells (A-F). ANGPT2 was localized in luteal cells and in interstitial cells, mostly those localized close to blood vessels (open arrowheads) expressing MHCII on consecutive sections, that were identified as macrophages (open arrowheads in E and F). No background staining is observed in the isotype control (inset in D).

were observed in luteal cells (Fig. 6A, B, C and D). Signals were detectable without striking differences throughout pregnancy, but appeared somewhat weaker at prepartum luteolysis (Fig. 6A, B, C and D).

\section{PGE2-mediated effects on expression of the ANGPT- system in canine primary luteal cells from early diestrus}

Whereas ANGPT1 expression in luteal cells was significantly $(P<0.0001)$ decreased in response to the PGE2 treatment (Fig. 7A), expression of ANGPT2 showed the opposite effect, being strongly upregulated $(P<0.0001)$ (Fig. 7B). The expression of TIE2 did not change significantly ( $P=0.1)$ (Fig. 7C); TIE1 expression was generally low in isolated cells and remained below the detection limit in most of the isolates in response to treatment with PGE2. 


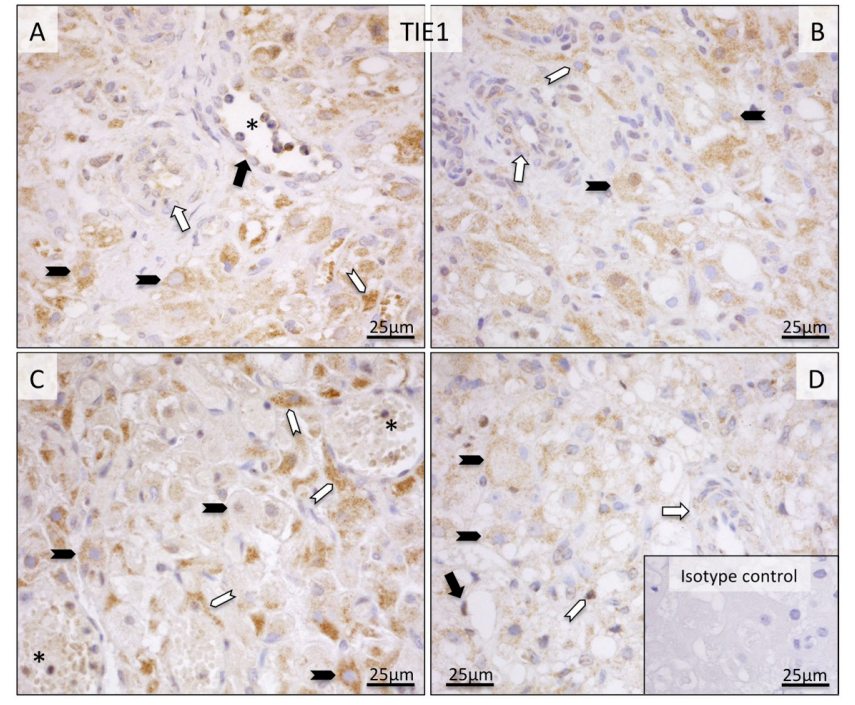

Figure 5 Immunohistochemical localization of TIE1 in canine $C L$ at selected time points throughout pregnancy: pre-implantation (A), post-implantation (B), mid-gestation (C) and prepartum luteolysis (D). TIE1 was expressed in the tunica media of the vessels (indicated as open arrows in A, B and D), endothelial cells (solid arrows in A and D), luteal cells (solid arrowheads in A, B, C and D) and in luteal macrophages (open arrowheads in A, B, C and D). Leukocytes stained with anti-TIE1 antibody are also localized in blood vessels (asterisks in A and C). No background staining is detected in the isotype control (insert to D).

\section{Discussion}

By interacting with other angiogenic and vasoactive factors, ANGPTs play crucial roles in balancing vascular maintenance and regression. Here, in addition to our previous studies showing time-dependent expression of VEGFA- and ET-family members, expression of the ANGPT-system was investigated in canine $C L$ at selected time points during pregnancy from the early luteal phase until prepartum luteolysis. Distinct expression and localization patterns were found for ANGPTs and their receptors. Thus, whereas ANGPT1 was found predominantly in tunica media of large blood vessels, and its mRNA showed a time-related expression pattern with higher expression levels observed in early $\mathrm{CL}$, ANGPT2 was predominantly localized in luteal cells, with no signals detectable in luteal vessels. Its signals were, however, clearly detectable throughout the CL life span. Interestingly, both factors, ANGPT1 and -2, were localized in interstitial cells found in close proximity to vessels, which were identified as macrophages (Nowaczyk et al. 2017). The increased availability of ANGPTs during the early luteal phase was associated with elevated levels of TIE1 receptor. TIE2 receptor was less strikingly regulated throughout the luteal phase, but appeared abundantly expressed, in particular in the vascular bed. The IHC signals appeared to be weaker during prepartum luteolysis, when TIE1 expression was

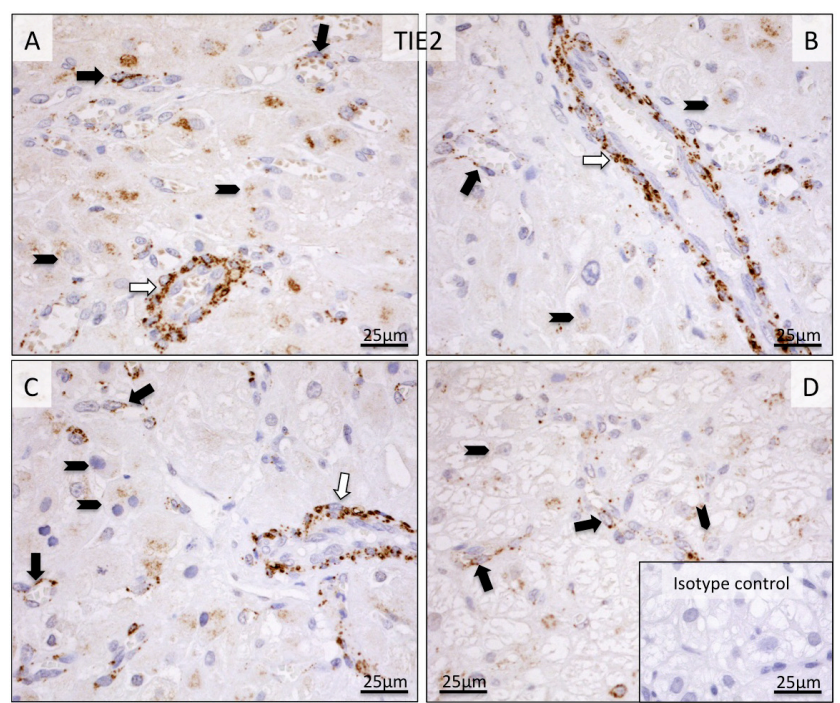

Figure 6 Localization of TIE2 in canine CL at selected time points throughout pregnancy: pre-implantation (A), post-implantation (B), mid-gestation (C) and prepartum luteolysis (D). TIE2 expression is observed in the tunica media of blood vessels (open arrows in A, B and $C$ ), endothelial cells (solid arrows in $A, B, C$ and $D$ ) and weaker signals in luteal cells (solid arrowheads in A, B, C and D). No background staining is seen in the isotype control (insert to D).

also decreased. Whereas the localization pattern of TIE1 appeared similar to the distribution of ANGPT2, and targeted mainly to luteal and immune cells, TIE2 receptor seemed to mirror the localization of ANGPT1 and was predominantly found in luteal vessels. These distribution patterns of ANGPT family members clearly indicate possible interplay between different cellular components of the CL and, thereby, an involvement of ANGPTs in local autocrine and paracrine regulatory mechanisms. Thus, acting through its receptors, in particular TIE2, the clearly detectable ANGPT2, together with increased expression of VEGFA at the beginning of the luteal phase (Gram et al. 2015a), could contribute to development of the canine CL by stimulating sprouting of blood vessels. This would resemble the mechanism described before in humans (Wulff et al. 2000). Furthermore, the ANGPT1-mediated (Shirasuna et al. 2012) recruitment of periendothelial cells (i.e., pericytes) in the presence of VEGFA is a phenomenon described before for some species, e.g., for cattle (Schams \& Berisha 2004), buffalo (Mishra et al. 2016) and rats (Matsuoka-Sakata et al. 2006). This could also apply in the canine species in which elevated VEGFA at post implantation (Gram et al. 2015a) coincides with the clearly detectable presence of ANGPT1 and TIE2 receptor in luteal vessels, possibly cumulatively contributing to structural and functional stabilization of a fully developed vascular bed. Later during pregnancy, at mid-gestation, a significant decrease in VEGFA expression (Gram et al. 2015a), at still relatively high availability of ANGPT1, could result in suppression of vascular function. Moreover, with 

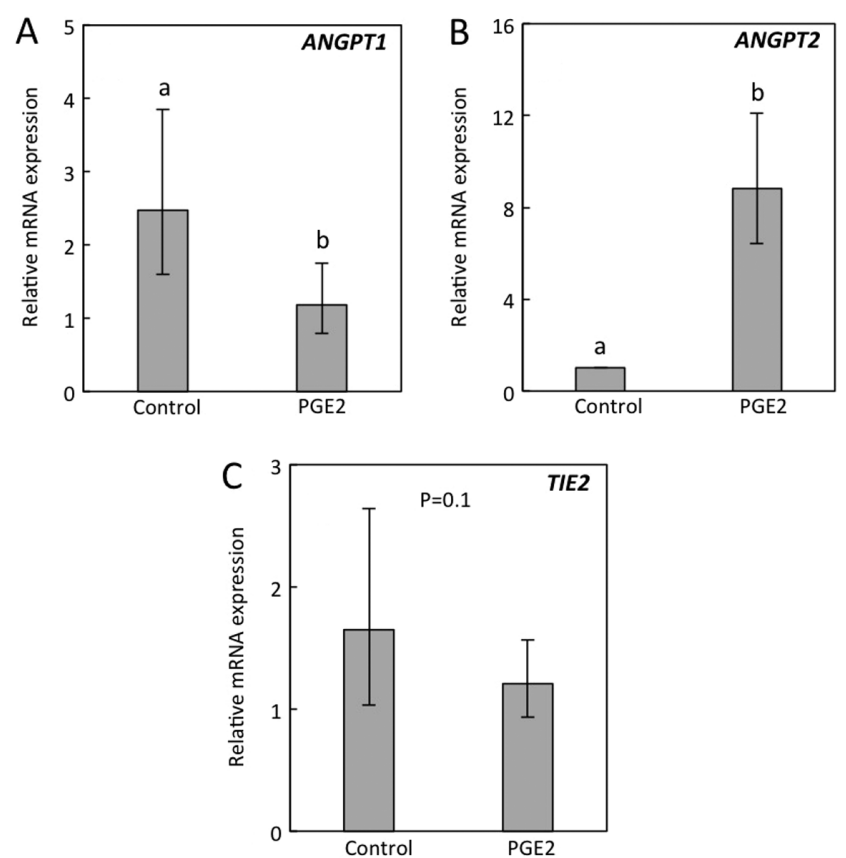

Figure 7 Effects of PGE2 on mRNA expression of ANGPT1, ANGPT2 and TIE2 in canine primary luteal cells obtained from non-pregnant dogs during the early luteal phase. Non-stimulated cells served as control. ANGPT1 (A), ANGPT2 (B) and TIE2 (C) mRNA expression was determined by real-time (TaqMan) PCR (Xg \pm S.D.). Bars with different letters differ at $P<0.0001$.

regard to vascular function, ANGPTs were described as being involved in the regulation of vascular tone and blood flow, acting on smooth muscle cells (Alfieri et al. 2014), in which canine ANGPT1 and TIE2 were also localized. Similarly, ANGPT-mediated nitric oxide (NO) release in arterioles was shown previously in mice (Alfieri et al. 2014). Nevertheless, the involvement of ANGPTs in regulating luteal vascular tone and blood flow in the dog remains to be elucidated. Furthermore, in particular, the localization of TIE1 and ANGPT2 in luteal cells, suggests involvement of ANGPTs in the regulation of luteal cell function. This seems to be an interesting finding; however, the exact function of ANGPTs in steroidogenic cells remains to be elucidated.

Although the physiological relevance of immune cells in regulating canine luteal function is not fully explored, the expression and relatively high abundance of the ANGPT system, mainly of ANGPT2 and TIE1, in immune cells attracted our attention. Based on our recent report, and as indicated elsewhere, these cells were identified as macrophages (Nowaczyk et al. 2017). In that previous research, the expression and localization of CD4-, CD8and MHC II-positive cells were investigated in canine CL of pregnancy (Nowaczyk et al. 2017). While CD4and CD8-positive macrophages were most abundantly represented during early luteal development, $\mathrm{MHCII}$ was elevated during luteolysis (Nowaczyk et al. 2017). The significance of immune cells, i.e., macrophages, eosinophils and neutrophils in developing $\mathrm{CL}$ and their possible implication in vascular function, was shown in mice, in which depletion of luteal macrophages resulted in insufficient P4 production and disruption of the luteal microvascular network (Care et al. 2013). With regard to ANGPTs, there is evidence showing that ANGPT2 is capable of promoting leukocyte attachment to endothelial cells by activating expression of adhesion molecules such as intercellular adhesion molecule 1 (ICAM1) and vascular cell adhesion molecule 1 (VCAM1) (Fiedler et al. 2006). Therefore, ANGPTs seem not only to exhibit direct angiogenic functions but also appear to be indirectly involved in luteal angiogenesis and vasculogenesis by regulating infiltration of immune cells (Fiedler et al. 2006). Whether this applies in the canine species, and the extent to which ANGPTs and their receptors could be involved in immune systemmediated regulation of canine luteal function, remains to be elucidated.

In developing canine $\mathrm{CL}$, PGE2 seems to be among the important luteotrophic factors (Kowalewski et al. 2013). As shown in our previous study, the direct effects of PGE2 in regulating canine CL function relate to stimulation of steroidogenic acute regulatory (STAR) protein expression and steroid synthesis in luteal cells (Kowalewski et al. 2013). Even more peculiar appear to be the apparent indirect effects of PGE2 in the canine $\mathrm{CL}$ shown in vitro by our group in studies utilizing isolated luteal cells (Gram et al. 2015b, Kowalewski et al. 2015). Thus, PGE2 was shown to enhance the expression of prolactin receptor (PRLR) (Kowalewski et al. 2015) and ETB (Gram et al. 2015b) in these cells. Here, by applying the same in vitro approach, PGE2 was shown to be capable of modulating expression of the ANGPT system. Whereas ANGPT1 mRNA was downregulated, the expression of ANGPT2, which was highly represented in canine luteal cells, was further potentiated by PGE2. In accordance with our IHC studies, showing relatively weak expression of TIE 1 and TIE2 in canine luteal cells, the expression of both receptors either did not change (TIE2) in response to PGE2 or was very low or even below the detection limit (TIE1). Taking into account the luteotropic role of PGE2 during the early luteal phase in the dog, and the aforementioned concomitantly increased expression of VEGFA (Gram et al. 2015a), the role of PGE2 in promoting angiogenesis through upregulation of ANGPT2 expression is implied and certainly merits further research. Similarly, the interplay between luteal cell-derived ANGPTs and vascular function is worth more attention. Cumulatively, with regard to PGE2, our findings add a new aspect to the indirect regulatory functions of PGE2 in regulating canine $\mathrm{CL}$, namely through induction of expression of vasoactive and angiogenic factors from the ANGPT family. The possible translational aspects of these findings should also be investigated in other species. 


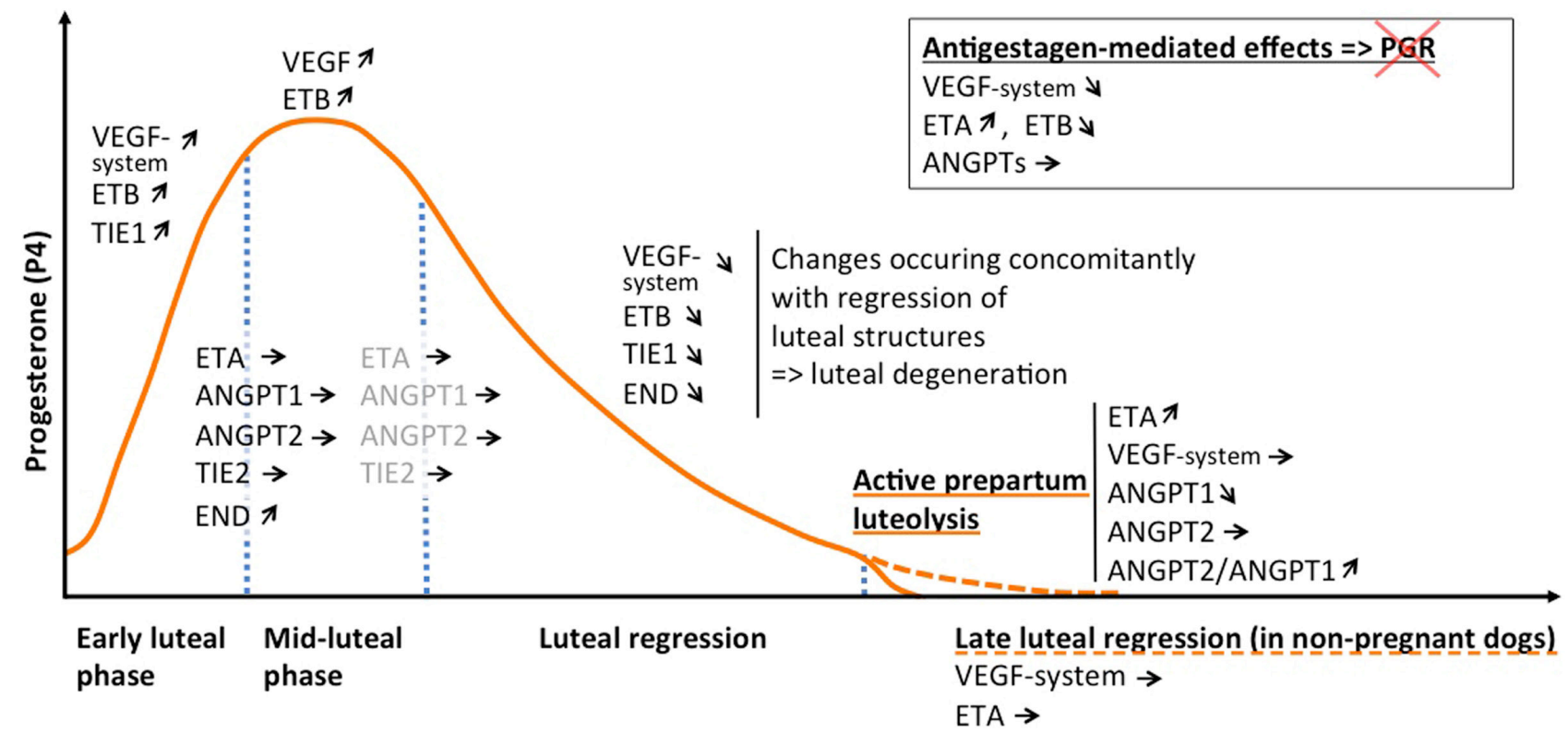

Figure 8 Cumulative schematic representation of expression profiles of angiogenic (VEGF system, endoglin, ANGTP system) and vasoactive (endothelin receptor B (ETB) and ETA) factors in the CL of pregnant and non-pregnant dogs. The expression of ANGPT system relates to the herein investigated $\mathrm{CL}$ of pregnant dogs. Arrows indicate the expression patterns of each factor: upregulation/increased expression $\nearrow$; downregulation/decreased expression \; or unchanged expression $\rightarrow$. The early- and mid-luteal phases are characterized by increased blood supply and high vascularization rate as indicated by increased availability of the VEGF system and vasodilatory endothelin receptor B (ETB) and increased presence of endoglin (END). During luteal regression, vascularization of the CL is slowed down and does not change strongly during the extended luteal regression observed in non-pregnant cycles. Conversely, in pregnant dogs, an increased expression of the vasoconstrictive endothelin receptor (ETA) and elevated ANGPT2/ANGPT1 ratio are observed during prepartum luteolysis. No such increase in ETA is observed during late luteal regression in non-pregnant dogs. While blocking the progesterone receptor (PGR) does not affect the expression of ANGPT family members, the expression of ETA is upregulated, and luteal levels of ETB and the VEGF-system decrease.

Cessation of the luteal phase in pregnant dogs is associated with increased expression of vasoconstrictive ETA during active prepartum luteolysis, implying involvement of PGF2 $\alpha$ in this process (Nohr et al. 1993, Gram et al. 2015b). In this context, an additional regulatory aspect arises from assessing the ANGPT2/ ANGPT1 ratio in our study. As implied, e.g., in cattle (Goede et al. 1998) and mice (Maisonpierre et al. 1997), during luteolysis, the increased ratio of ANGPT2/ ANGPT1 seems to play an important role in vascular regression via inducing loosening of periendothelial support and initializing apoptosis of endothelial cells. Based on the observations from the present study, this may also apply in the canine species, in which upregulation of the ANGPT2/ANGPT1 ratio during natural prepartum luteolysis in the absence of VEGFA implies destabilization of blood vessels.

Possible P4-mediated effects on ANGPT-system expression were investigated in experiments in which mid-pregnant dogs were treated with an antigestagen, aglepristone. This targeted suppression of P4 receptor (PGR) did not, however, affect the expression of ANGPT family members in our study. This contrasts with the effects aglepristone exerts on the expression of other angiogenic and vasoactive factors, which are strongly regulated following treatment. Thus, whereas the expression of vasoconstrictive ETA was induced, the expression of ETB and VEGF family members (VEGF 164, VEGFR1 and VEGFR2), was significantly suppressed (Kowalewski 2014, Gram et al. 2015b). Therefore, it appears plausible that PGR is not involved in the angiogenic and vasoactive effects exerted by the ANGPT system in the canine CL.

Cumulatively, our study adds important information and complements our previous reports addressing the possible functions of the ET and VEGF systems in the dog. A summary of the most important findings from our current and previous studies, depicting the proposed model of involvement of the vascular system in regulating canine luteal function, is presented in Fig. 8.

With regard to the results presented herein, by combining a descriptive study with functional in vivo and in vitro approaches, the expression and potential roles of the ANGPT-system have been investigated in canine $\mathrm{CL}$ during pregnancy. Based on our results, ANGPTs appear to be involved in both vasoactive and angiogenic functions in the dog. Among the most important findings is the ubiquitous expression of ANGPTs in different cellular components of the canine $\mathrm{CL}$ throughout pregnancy. This includes luteal cells, the vascular bed and, importantly, immune system-derived cells. Whereas P4 is the most important luteal hormone 
and its production depends on adequate vascularization of the $\mathrm{CL}$, it does not seem to directly support the expression of ANGPTs. The modulatory effect that PGE2 exerts on ANGPTs expression in luteal cells is certainly a new aspect with considerable translational importance, worthy of more detailed studies in the future.

\section{Declaration of interest}

The authors declare that there is no conflict of interest that could be perceived as prejudicing the impartiality of the research reported.

\section{Funding}

This research did not receive any specific grant from any funding agency in the public, commercial or not-for-profit sector.

\section{Author contribution statement}

A G: Concept of the study, experimental design, generating data, analysis and interpretation of data and writing of the manuscript. M T P: Involved in the laboratory part of the project. A B, A T G-B, M P K: Knowledge transfer, critical discussion of data, editing of the manuscript.

\section{Acknowledgements}

Authors are thankful to Dr Barry Bavister for careful editing of the manuscript. They are also grateful to Prof. Dr Selim Aslan, University of Ankara, Turkey, and Prof. Dr Bernd Hoffmann, Justus-Liebig University, Giessen, and their teams for the provision of the tissue material. The technical expertise and contributions of Elisabeth Högger and Ricardo Fernandez Rubia are greatly appreciated. Part of the laboratory work was performed using the logistics at the Center for Clinical Studies, Vetsuisse Faculty, University of Zurich.

\section{References}

Alfieri A, Ong AC, Kammerer RA, Solanky T, Bate S, Tasab M, Brown NJ \& Brookes ZL 2014 Angiopoietin-1 regulates microvascular reactivity and protects the microcirculation during acute endothelial dysfunction: role of eNOS and VE-cadherin. Pharmacological Research $8043-51$. (https:// doi.org/10.1016/j.phrs.2013.12.008)

Brindle NP, Saharinen P \& Alitalo K 2006 Signaling and functions of angiopoietin-1 in vascular protection. Circulation Research 98 1014-1023. (https://doi.org/10.1161/01.RES.0000218275.54089.12)

Care AS, Diener KR, Jasper MJ, Brown HM, Ingman WV \& Robertson SA 2013 Macrophages regulate corpus luteum development during embryo implantation in mice. Journal of Clinical Investigation 123 3472-3487. (https://doi.org/10.1172/JCl60561)

Cebe-Suarez S, Zehnder-Fjallman A \& Ballmer-Hofer K 2006 The role of VEGF receptors in angiogenesis; complex partnerships. Cellular and Molecular Life Sciences 63 601-615. (https://doi.org/10.1007/s00018005-5426-3)

Channing CP, Schaerf FW, Anderson LD \& Tsafriri A 1980 Ovarian follicular and luteal physiology. International Review of Physiology 22 117-201.
Concannon PW, McCann JP \& Temple M 1989 Biology and endocrinology of ovulation, pregnancy and parturition in the dog. Journal of Reproduction and Fertility Supplements 39 3-25.

Felcht M, Luck R, Schering A, Seidel P, Srivastava K, Hu J, Bartol A, Kienast Y, Vettel C, Loos EK et al. 2012 Angiopoietin-2 differentially regulates angiogenesis through TIE2 and integrin signaling. Journal of Clinical Investigation 122 1991-2005. (https://doi.org/10.1172/ JCI58832)

Ferrara N \& Davis-Smyth T 1997 The biology of vascular endothelial growth factor. Endocrine Reviews 18 4-25. (https://doi.org/10.1210/ edrv.18.1.0287)

Fiedler U, Reiss Y, Scharpfenecker M, Grunow V, Koidl S, Thurston G, Gale NW, Witzenrath M, Rosseau S, Suttorp N et al. 2006 Angiopoietin-2 sensitizes endothelial cells to TNF-alpha and has a crucial role in the induction of inflammation. Nature Medicine 12 235-239. (https://doi. org/10.1038/nm1351)

Fraser HM \& Wulff C 2003 Angiogenesis in the corpus luteum. Reproductive Biology and Endocrinology 1 88. (https://doi.org/10.1186/1477-7827-188)

Goede V, Schmidt T, Kimmina S, Kozian D \& Augustin HG 1998 Analysis of blood vessel maturation processes during cyclic ovarian angiogenesis. Laboratory Investigation 78 1385-1394.

Gram A, Hoffmann B, Boos A \& Kowalewski MP 2015a Expression and localization of vascular endothelial growth factor A (VEGFA) and its two receptors (VEGFR1/FLT1 and VEGFR2/FLK1/KDR) in the canine corpus luteum and utero-placental compartments during pregnancy and at normal and induced parturition. General and Comparative Endocrinology 223 54-65. (https://doi.org/10.1016/j.ygcen.2015.09.020)

Gram A, Latter S, Boos A, Hoffmann B \& Kowalewski MP 2015b Expression and functional implications of luteal endothelins in pregnant and nonpregnant dogs. Reproduction 150 405-415. (https://doi.org/10.1530/ REP-15-0256)

Hanahan D 1997 Signaling vascular morphogenesis and maintenance. Science 277 48-50. (https://doi.org/10.1126/science.277.5322.48)

Hazzard TM \& Stouffer RL 2000 Angiogenesis in ovarian follicular and luteal development. Baillière's Best Practice and Research in Clinical Obstetrics and Gynaecology 14 883-900. (https://doi.org/10.1053/ beog.2000.0133)

Hoffmann B, Hoveler R, Nohr B \& Hasan SH 1994 Investigations on hormonal changes around parturition in the dog and the occurrence of pregnancy-specific non conjugated oestrogens. Experimental and Clinical Endocrinology 102 185-189. (https://doi.org/10.1055/s-0029-1211280)

Hoffmann B, Busges F \& Baumgartner W 2004a Immunohistochemical detection of CD4-, CD8- and MHC II-expressing immune cells and endoglin in the canine corpus luteum at different stages of dioestrus. Reproduction in Domestic Animals 39 391-395. (https://doi. org/10.1111/j.1439-0531.2004.00520.x)

Hoffmann B, Busges F, Engel E, Kowalewski MP \& Papa P $2004 b$ Regulation of corpus luteum-function in the bitch. Reproduction in Domestic Animals 39 232-240. (https://doi.org/10.1111/j.1439-0531.2004.00508.x)

Janowski T, Fingerhut J, Kowalewski MP, Zdunczyk S, Domoslawska A, Jurczak A, Boos A, Schuler G \& Hoffmann B 2014 In vivo investigations on luteotropic activity of prostaglandins during early diestrus in nonpregnant bitches. Theriogenology 82 915-920. (https://doi. org/10.1016/j.theriogenology.2014.07.005)

Kautz E, Gram A, Aslan S, Ay SS, Selcuk M, Kanca H, Koldas E, Akal E, Karakas K, Findik M et al. 2014 Expression of genes involved in the embryo-maternal interaction in the early-pregnant canine uterus. Reproduction 147 703-717. (https://doi.org/10.1530/REP-13-0648)

Kowalewski MP 2014 Luteal regression vs prepartum luteolysis: regulatory mechanisms governing canine corpus luteum function. Reproductive Biology 14 89-102. (https://doi.org/10.1016/j.repbio.2013.11.004)

Kowalewski MP, Mason JI, Howie AF, Morley SD, Schuler G \& Hoffmann B 2006a Characterization of the canine 3beta-hydroxysteroid dehydrogenase and its expression in the corpus luteum during diestrus. Journal of Steroid Biochemistry and Molecular Biology $101254-262$. (https://doi.org/10.1016/j.jsbmb.2006.06.029)

Kowalewski MP, Schuler G, Taubert A, Engel E \& Hoffmann B $2006 b$ Expression of cyclooxygenase 1 and 2 in the canine corpus luteum during diestrus. Theriogenology 66 1423-1430. (https://doi.org/10.1016/j. theriogenology.2006.01.039) 
Kowalewski MP, Meyer A, Hoffmann B, Aslan S \& Boos A 2011 a Expression and functional implications of peroxisome proliferatoractivated receptor gamma (PPARgamma) in canine reproductive tissues during normal pregnancy and parturition and at antiprogestin induced abortion. Theriogenology 75 877-886. (https://doi.org/10.1016/j. theriogenology.2010.10.030)

Kowalewski MP, Michel E, Gram A, Boos A, Guscetti F, Hoffmann B, Aslan S \& Reichler I 2011b Luteal and placental function in the bitch: spatio-temporal changes in prolactin receptor (PRLr) expression at dioestrus, pregnancy and normal and induced parturition. Reproductive Biology and Endocrinology 9 109. (https://doi.org/10.1186/1477-78279-109)

Kowalewski MP, Fox B, Gram A, Boos A \& Reichler I 2013 Prostaglandin E2 functions as a luteotrophic factor in the dog. Reproduction 145 213-226. (https://doi.org/10.1530/REP-12-0419)

Kowalewski MP, Ihle S, Siemieniuch MJ, Gram A, Boos A, Zdunczyk S, Fingerhut J, Hoffmann B, Schuler G, Jurczak A et al. 2015 Formation of the early canine $\mathrm{CL}$ and the role of prostaglandin E2 (PGE2) in regulation of its function: an in vivo approach. Theriogenology 83 1038-1047. (https://doi.org/10.1016/j.theriogenology.2014.12.006)

Maisonpierre PC, Suri C, Jones PF, Bartunkova S, Wiegand SJ, Radziejewski C, Compton D, McClain J, Aldrich TH, Papadopoulos N et al. 1997 Angiopoietin-2, a natural antagonist for Tie2 that disrupts in vivo angiogenesis. Science 277 55-60. (https://doi.org/10.1126/ science.277.5322.55)

Matsuoka-Sakata A, Tamura H, Asada H, Miwa I, Taketani T, Yamagata Y \& Sugino N 2006 Changes in vascular leakage and expression of angiopoietins in the corpus luteum during pregnancy in rats. Reproduction 131 351-360. (https://doi.org/10.1530/rep.1.00947)

Mishra SR, Parmar MS, Yadav VP, Reshma R, Bharati J, Bharti MK, Paul A, Chouhan VS, Taru Sharma G, Singh G et al. 2016 Expression and localization of angiopoietin family in corpus luteum during different stages of oestrous cycle and modulatory role of angiopoietins on steroidogenesis, angiogenesis and survivability of cultured buffalo luteal cells. Reproduction in Domestic Animals 51 855-869. (https://doi. org/10.1111/rda.12739)

Nishiyama T, Tsumagari S, Ito M, Kimura J, Watanabe G, Taya K \& Takeishi M 1999 Immunohistochemical study of steroidogenic enzymes in the ovary and placenta during pregnancy in the dog. Anatomia, Histologia, Embryologia 28 125-129. (https://doi.org/10.1046/j.14390264.1999.00170.x)

Nohr B, Hoffmann B \& Steinetz BE 1993 Investigation of the endocrine control of parturition in the dog by application of an antigestagen. Journal of Reproduction and Fertility Supplements 47 542-543.
Nowaczyk RM, Jursza-Piotrowska E, Gram A, Siemieniuch MJ, Boos A \& Kowalewski MP 2017 Cells expressing CD4, CD8, MHCII and endoglin in the canine corpus luteum of pregnancy, and prepartum activation of the luteal TNFalpha system. Theriogenology 98 123-132. (https://doi. org/10.1016/j.theriogenology.2017.05.003)

Schams D \& Berisha B 2004 Regulation of corpus luteum function in cattle - an overview. Reproduction in Domestic Animals 39 241-251. (https:// doi.org/10.1111/j.1439-0531.2004.00509.x)

Shirasuna K, Nitta A, Sineenard J, Shimizu T, Bollwein H \& Miyamoto A 2012 Vascular and immune regulation of corpus luteum development, maintenance, and regression in the cow. Domestic Animal Endocrinology 43 198-211. (https://doi.org/10.1016/j.domaniend.2012.03.007)

Smith MF, McIntush EW \& Smith GW 1994 Mechanisms associated with corpus luteum development. Journal of Animal Science 72 1857-1872. (https://doi.org/10.2527/1994.7271857x)

Stouffer RL \& Hannebold JD 2015 Structure, function, and regulation of the corpus luteum. In The Knobil and Neill's Physiology of Reproduction, pp 1023-1076.

Stuttfeld E \& Ballmer-Hofer K 2009 Structure and function of VEGF receptors. IUBMB Life 61 915-922. (https://doi.org/10.1002/iub.234)

Sugino N, Suzuki T, Sakata A, Miwa I, Asada H, Taketani T, Yamagata Y \& Tamura H 2005 Angiogenesis in the human corpus luteum: changes in expression of angiopoietins in the corpus luteum throughout the menstrual cycle and in early pregnancy. Journal of Clinical Endocrinology and Metabolism 90 6141-6148. (https://doi.org/10.1210/jc.2005-0643)

Wulff C, Wilson H, Largue P, Duncan WC, Armstrong DG \& Fraser HM 2000 Angiogenesis in the human corpus luteum: localization and changes in angiopoietins, tie-2, and vascular endothelial growth factor messenger ribonucleic acid. Journal of Clinical Endocrinology and Metabolism 85 4302-4309.

Zatta S, Rehrauer H, Gram A, Boos A \& Kowalewski MP 2017 Transcriptome analysis reveals differences in mechanisms regulating cessation of luteal function in pregnant and non-pregnant dogs. BMC Genomics 18757. (https://doi.org/10.1186/s12864-017-4084-9)

Received 4 May 2018

First decision 15 June 2018

Revised manuscript received 11 July 2018

Accepted 2 August 2018 